Mathematical Modelling and Analysis

Volume 10 Number 4, 2005, PAGES 353-366

(c) 2005 Technika ISSN 1392-6292

\title{
PERONA-MALIK EQUATION - ERROR ESTIMATES FOR EXPLICIT FINITE VOLUME SCHEME
}

A. HANDLOVIČOVÁ and Z. KRIVÁ

Department of Mathematics, Slovak University of Technology

Radlinského 11, 81368 Bratislava, Slovakia

E-mail: angela@math.sk, kriva@math.sk

Received April 25 2005; revised October 212005

\begin{abstract}
Error estimates in the $L_{2}$ norm for the explicit fully discrete numerical finite volume scheme are derived and proved for Perona-Malik equation. Numerical example is also presented.

Key words: time explicit numerical scheme, Perona-Malik equation, image processing, finite volume method, error estimates
\end{abstract}

\section{Introduction}

\subsection{Mathematical model of the problem}

Perona-Malik problem discussed in [3] has the following form

$$
\begin{aligned}
& \partial_{t} u-\nabla \cdot\left(g\left(\left|\nabla G_{\sigma} * u\right|\right) \nabla u\right)=0 \quad \text { in } Q_{T} \equiv I \times \Omega, \\
& \partial_{\nu} u=0 \quad \text { on } I \times \partial \Omega, \\
& u(0, \cdot)=u_{0} \quad \text { in } \Omega,
\end{aligned}
$$

where $\Omega \subset \mathbb{R}^{d}$ is a rectangular domain, $I=[0, T]$ is a scaling interval, $g(s)$ is a Lipschitz continuous decreasing function with Lipschitz constant $L_{g}$,

$$
g(0)=1, \quad 0<g(s) \rightarrow 0 \text { for } s \rightarrow \infty
$$

$G_{\sigma} \in C^{\infty}\left(\mathbb{R}^{d}\right)$ is a smoothing kernel with compact support $K_{\sigma}$, such that

$$
\int_{\mathbb{R}^{d}} G_{\sigma}(x) d x=1, \quad G_{\sigma}(x) \rightarrow \delta_{x} \quad \text { for } \sigma \rightarrow 0,
$$

$\delta_{x}$ is the Dirac function at point $x$, initial condition $u_{0}$ is such that regularity stated below is fulfilled. 
We can rewrite the partial differential equation (1.1) in the form

$$
\partial_{t} u-\nabla \cdot(g(|J(u)(x)|) \nabla u)=0 \quad \text { in } Q_{T} \equiv I \times \Omega,
$$

where $J(u): L_{2}(\Omega) \rightarrow\left(C^{\infty}(\Omega)\right)^{d}$. In our case we use $J(u)(t, \cdot)=\nabla G_{\sigma} * u(t, \cdot)$ for $t$ fixed, but we can choose any smoothing operator with these properties.

Let us define a weak solution of the problem (1.4),(1.2),(1.3). Equation (1.4) is multiplied by a test function $\varphi \in \Psi$, where $\Psi$ is the space of smooth test functions

$$
\Psi=\left\{\varphi \in C^{1,2}([0, T] \times \bar{\Omega}), \nabla \varphi \cdot \vec{n}=0 \text { on }(0, T) \times \partial \Omega, \varphi(T, \cdot)=0\right\} .
$$

Integrating over $[0, T] \times \Omega$ and applying integration by parts and properties of a test function, we come to a definition of the weak solution.

Definition 1. The weak solution of the regularized Perona-Malik problem (1.1)-(1.3) is a function $\mathrm{u} \in L_{2}\left(I, W^{1,2}(\Omega)\right)$ satisfying the identity

$$
\begin{aligned}
\int_{0}^{T} \int_{\Omega} u \frac{\partial \varphi}{\partial t}(t, x) d x d t & +\int_{\Omega} u_{0}(x) \varphi(0, x) d x \\
& -\int_{0}^{T} \int_{\Omega} g(|J(u(t, x))|) \nabla u(t, x) \nabla \varphi(t, x) d x d t=0
\end{aligned}
$$

for all $\varphi \in \Psi$.

It is well known from the regularity theory of such solutions [6] that, due to given properties of the operator $J(u)$, the weak solution of our problem is a function $u \in L_{2}\left(I, W^{2,2}(\Omega)\right)$ for initial condition $u_{0} \in L_{\infty}(\Omega)$. Moreover it follows from the embedding theory for dimensions $d=2$ and $d=3$ that $u \in C(\bar{\Omega})$ for almost all $t \in I$. To obtain our error estimates we need further regularity of the solution, more precisely $u \in L_{2}\left(I, W^{2,2}(\Omega)\right) \cap L_{\infty}\left(I, W^{1,2}(\Omega)\right)$, $\partial_{t t} u \in L_{1}\left(I, L_{1}(\Omega)\right)$ and $\nabla\left(\partial_{t} u\right) \in L_{1}\left(I, L_{1}(\Omega)\right)$.

\subsection{Formulation of the finite volume scheme}

Let $\tau_{h}$ be a uniform mesh covering $\Omega$ with cells $p$ of measure $m(p)$ (we assume rectangular cells here). For every cell $p$ we consider a set of its neighbours $N(p)$ consisting of all cells $q \in \tau_{h}$ for which the common interface of $p$ and $q$, denoted by $e_{p q}$, is of non-zero measure $m\left(e_{p q}\right)$. We denote the set of all these edges for all volumes $p \in \tau_{h}$ by $\mathcal{E}$ and by $e_{p q} I$ we denote the edge which connects the volumes $p$ and $q$ (clearly $e_{p q}=e_{q p}=e_{p q} I$ ).

It is assumed that for every $p$, there exists a representative point $x_{p} \in p$, such that for every pair $p, q, q \in N(p)$, the vector $\frac{x_{q}-x_{p}}{\left|x_{q}-x_{p}\right|}$ is equal to a unit vector $n_{p q}$ which is normal to $e_{p q}$ and oriented from $p$ to $q$. We denote $d_{p q}:=$ 
$\left|x_{p}-x_{q}\right|$. In a simple case of a uniform grid $x_{p}$ is just the center of the pixel. Then, let $x_{p q}$ be the point of $e_{p q}$ intersecting the segment $\overline{x_{p} x_{q}}$. We define

$$
T_{p q}:=\frac{m\left(e_{p q}\right)}{d_{p q}}
$$

Moreover there exist constants $\underline{T}, \bar{T}$, such that for all mesh $\tau_{h}$ it holds $\underline{T} \leq T_{p q} \leq \bar{T}$.

Remark. It is obvious that if we consider a uniform mesh, there are only several types of the values for $d_{p q}, T_{p q}$ and $m\left(e_{p q}\right)$. For example if all volumes are squares, then $d_{p q}, T_{p q}$ and $m\left(e_{p q}\right)$ have the same value for all volumes, for rectangles they have two different values etc. We will use the notation introduced above in accordance with the notation used in the cited articles.

Discrete approximation of a solution of partial differential equation is considered to be piecewise constant on control volumes ([4]). Let $u_{p}^{n}$ be the value of the numerical solution in the $n$-th scale step on a volume $p$. The finite volume explicit scheme is then written as follows:

Let $0=t_{0} \leq t_{1} \leq \ldots \leq t_{n} \ldots \leq t_{N_{\max }}, \quad N_{\max } k=T$ denote the scale discretization steps with $t_{l}=t_{l-1}+k$, where $k$ is the discrete scale step, $l=1,2, \ldots N_{\max }$. For $n=0, \ldots, N_{\max }-1$ we look for $u_{p}^{n+1}, p \in \tau_{h}$, satisfying the identities

$$
\begin{aligned}
& \left(u_{p}^{n+1}-u_{p}^{n}\right) m(p)=k \sum_{q \in N(p)} g_{p q}^{\sigma, n} T_{p q}\left(u_{q}^{n}-u_{p}^{n}\right), \\
& u_{p}^{0}=\frac{1}{m(p)} \int_{p} u_{0}(x) d x, \quad g_{p q}^{\sigma, n}:=g\left(\left|J\left(\tilde{u}\left(t_{n}, x_{p q}\right)\right)\right|\right),
\end{aligned}
$$

where $\tilde{u}$ is a periodic extension of the discrete image computed in the $n$-th scale step. For computation details see [1].

Its $L_{2}$ norm can be estimated with constant $B$ by $L_{2}$ norm of the function $u$. $u_{p}^{n}$ is a value of the numerical solution on the volume $p$ in the $n$-th scale step. Let us denote by $\bar{u}_{h, k}$ the finite volume numerical solution for some fixed space and scale mesh $h$ and $k$. This solution is piecewise constant on each finite volume and in each scale step as is usual for finite volume numerical schemes of a parabolic type. By $\bar{u}^{l}$ we denote the function piecewise constant on each finite volume in the $l$-th scale step.

\subsection{Previous results}

In [2] error estimates for similar but implicit numerical scheme based on the finite volume space discretization were proved in the form:

Theorem 1. Let the relation between scale and space discretization is given as follows $k=C h$. Then the following error estimates hold for Perona-Malik weak solution and numerical solution obtained via finite volume method 


$$
\sum_{n=0}^{N_{\max }} \int_{I_{n}} \int_{\Omega}\left|u\left(t_{n+1}, x\right)-\bar{u}_{h, k}\left(t_{n+1}, x\right)\right|^{2} d x d t \leq C h
$$

and

$$
\sum_{n=0}^{m-1} \int_{I_{n}} \sum_{e_{p q} I} m\left(e_{p q}\right) d_{p q}\left(\frac{u_{q}^{n+1}-u_{p}^{n+1}}{d_{p q}}-\frac{1}{m\left(e_{p q}\right)} \int_{e_{p q}} \nabla u \cdot \mathbf{n}_{\mathbf{p q}} d s\right)^{2} d t \leq C h .
$$

Similar results will be now proved also for the explicit numerical scheme.

\section{Stability and Convergence Results}

We briefly mention results of Krivá obtained for the explicit finite volume scheme concerning the stability and convergence properties for explicit finite volume numerical scheme for the Perona - Malik problem (see [1]). We need these results to prove error estimates for numerical method presented in this article. First we introduce the following stability assumption:

$$
k \leq(1-\xi) \frac{m(p)}{\sum_{q \in N(p)} g_{p q}^{\sigma, n} T_{p q}} \quad \text { for all } p \in \tau_{h} \text { and } \xi \in(0,1)
$$

Under this stability condition stability estimates for the explicit scheme are proved in [1] and for the semi-implicit scheme they are derived in [5]. These estimates are of the following type:

Lemma 1. (A priori estimates in $L_{2}\left(Q_{T}\right)$ ) There exist positive constants $C_{1}, C_{2}$ which do not depend on the $h, k$, such that

$$
\begin{aligned}
& \text { (i) } \max _{0 \leq l \leq N_{\max }} \sum_{p \in \tau_{h}}\left(u_{p}^{l}\right)^{2} m(p) \leq C_{1}, \\
& \text { (ii) } \sum_{l=0}^{N_{\max }} k \sum_{(p, q) \in \mathcal{E}} \frac{\left(u_{p}^{l}-u_{q}^{l}\right)^{2}}{d_{p q}} m\left(e_{p q}\right) \leq C_{2} .
\end{aligned}
$$

Lemma 2. (Convergence of $\bar{u}_{h, k}$.) There exists $u \in L^{2}\left(Q_{T}\right)$, which is the weak solution of (1.5) such that $\bar{u}_{h, k} \rightarrow u$ in $L^{2}\left(Q_{T}\right)$ as $h, k \rightarrow 0$. Furthermore, the convergence is pointwise.

\section{Error Estimates}

\section{1. $L_{\infty}$ stability for a discrete solution}

We rewrite the original discrete equation (1.6) in the following way: 


$$
u_{p}^{n+1}=u_{p}^{n}\left(1-\frac{k}{m(p)} \sum_{q \in N(p)} g_{p q}^{\sigma, n} T_{p q}\right)+\frac{k}{m(p)} \sum_{q \in N(p)} g_{p q}^{\sigma, n} T_{p q} u_{q}^{n} .
$$

From stability condition (2.1) we immediately can see, that both coefficients of $u_{p}^{n}$ and $u_{q}^{n}$ on the right hand side are positive. So, we have

$$
u_{p}^{n+1} \leq\left\|\bar{u}^{n}\right\|_{L_{\infty}(\Omega)}
$$

for all $p \in \tau_{h}$. Then it holds also:

$$
\left\|\bar{u}^{n+1}\right\|_{L_{\infty}(\Omega)} \leq\left\|\bar{u}^{n}\right\|_{L_{\infty}(\Omega)} .
$$

Recursively we get

$$
\left\|\bar{u}^{n+1}\right\|_{L_{\infty}} \leq\left\|\bar{u}^{0}\right\|_{L_{\infty}}=C .
$$

\subsection{Error estimates}

The method how to obtain the error estimates is similar to one given in [2], but there are some new terms and some terms must be estimated in different way so we describe the whole proof. Let now $t \in\left\langle t_{n}, t_{n+1}\right)$. Multiplying PDE (1.4) by $v_{p}^{n+1}$ and then integrating over volume $p$ and using integration by parts, we have:

$$
\int_{p} \partial_{t} u(t, x) v_{p}^{n+1} d x-\int_{\partial p} g(|J(u)|) \nabla u(t, x) \cdot \mathbf{n}_{\mathbf{p}} v_{p}^{n+1} d x=0,
$$

where $\partial p$ is the boundary of the volume $p$ and $\mathbf{n}_{\mathbf{p}}$ is the outward unit normal vector to the boundary of volume $p$ and further analogously $\mathbf{n}_{\mathbf{p q}}$ will be the outward unit normal vector to the edge $e_{p q}$. We can write $\partial p=\cup_{q \in N(p)} e_{p q}$. For the discrete scheme we have

$$
\frac{\left(u_{p}^{n+1}-u_{p}^{n}\right) v_{p}^{n+1} m(p)}{k}-\sum_{q \in N(p)} g_{p q}^{\sigma, n} T_{p q}\left(u_{q}^{n}-u_{p}^{n}\right) v_{p}^{n+1}=0 .
$$

Now we denote $e_{p}^{n}=u\left(t_{n}, x_{p}\right)-u_{p}^{n}$, where $x_{p}$ is a representative point of a volume $p, p \in \tau_{h}$.

Then posing $v_{p}^{n}=e_{p}^{n}$ and subtracting (3.4) from (3.3) we obtain:

$$
\begin{aligned}
\int_{p} \frac{\left(e_{p}^{n+1}-e_{p}^{n}\right)}{k} e_{p}^{n+1} d x & +\sum_{q \in N(p)} \int_{e_{p q}}\left(g_{p q}^{\sigma, n} \frac{u_{q}^{n}-u_{p}^{n}}{d_{p q}}-g(|J(u)|) \nabla u \cdot \mathbf{n}_{\mathbf{p q}}\right) e_{p}^{n+1} d x \\
= & \int_{p}\left(\frac{u\left(t_{n+1}, x_{p}\right)-u\left(t_{n}, x_{p}\right)}{k}-\partial_{t} u(t, x)\right) e_{p}^{n+1} d x .
\end{aligned}
$$

Now after summation over all volumes $p \in \tau_{h}$ and integration over $I_{n}=$ $\left\langle t_{n}, t_{n+1}\right)$ for all $n=0,1, \ldots, m-1$, where $m$ is arbitrary number from the set $\left\{1, \ldots, N_{\max }\right\}$ and rearranging the equation we obtain: 


$$
\begin{aligned}
& \int_{\Omega}\left|\bar{e}^{m}\right|^{2} d x+\sum_{n=0}^{m-1} \int_{\Omega}\left|\bar{e}^{n+1}-\bar{e}^{n}\right|^{2} d x+2 \sum_{n=0}^{m-1} \int_{I_{n}} \sum_{p \in \tau_{h}} \sum_{q \in N(p)} \\
& \times \int_{e_{p q}}\left(g_{p q}^{\sigma, n} \frac{u_{q}^{n}-u_{p}^{n}}{d_{p q}}-g(|J(u)|) \nabla u \cdot \mathbf{n}_{\mathbf{p q}}\right) e_{p}^{n+1} d x d t=\int_{\Omega}\left|\bar{e}^{0}\right|^{2} d x \\
& +2 \sum_{n=0}^{m-1} \int_{I_{n}} \sum_{p \in \tau_{h}} \int_{p}\left(\frac{u\left(t_{n+1}, x_{p}\right)-u\left(t_{n}, x_{p}\right)}{k}-\partial_{t} u\right) e_{p}^{n+1} d x d t .
\end{aligned}
$$

The third term on the left hand side of the last equation can be expressed as usually in the finite volume theory (see [4]):

$$
\begin{aligned}
& \text { "Third" }=2 \sum_{n=0}^{m-1} \int_{I_{n}} \sum_{p \in \tau_{h}} \sum_{q \in N(p)} \int_{e_{p q}}\left(g_{p q}^{\sigma, n} \frac{u_{q}^{n}-u_{p}^{n}}{d_{p q}}-g(|J(u)|) \nabla u \cdot \mathbf{n}_{\mathbf{p q}}\right) e_{p}^{n+1} d x d t \\
& =2 \sum_{n=0}^{m-1} \int_{I_{n}} \sum_{\mathcal{E}} \int_{e_{p q} I}\left(g_{p q}^{\sigma, n} \frac{u_{q}^{n}-u_{p}^{n}}{d_{p q}}-g(|J(u)|) \nabla u \cdot \mathbf{n}_{\mathbf{p q}}\right)\left(e_{p}^{n+1}-e_{q}^{n+1}\right) d x d t .
\end{aligned}
$$

After rearrangement we get:

$$
\begin{aligned}
& \text { "Third" }=2 \sum_{n=0}^{m-1} \int_{I_{n}} \sum_{\mathcal{E}} \int_{e_{p q} I} g(|J(u)|) \frac{\left(e_{p}^{n+1}-e_{q}^{n+1}\right)^{2}}{d_{p q}} d x d t \\
& +2 \sum_{n=0}^{m-1} \int_{I_{n}} \sum_{\mathcal{E}} \int_{e_{p q} I} g(|J(u)|) \frac{\left(e_{q}^{n+1}-e_{q}^{n}-\left(e_{p}^{n+1}-e_{p}^{n}\right)\right)}{d_{p q}}\left(e_{p}^{n+1}-e_{q}^{n+1}\right) d x d t \\
& +2 \sum_{n=0}^{m-1} \int_{I_{n}} \sum_{\mathcal{E}} \int_{e_{p q} I}\left(g_{p q}^{\sigma, n}-g(|J(u)|)\right) \frac{u_{q}^{n}-u_{p}^{n}}{d_{p q}}\left(e_{p}^{n+1}-e_{q}^{n+1}\right) d x d t+2 \sum_{n=0}^{m-1} \\
& \times \int_{I_{n}} \sum_{\mathcal{E}} \int_{e_{p q} I} g(|J(u)|)\left(\frac{u\left(t_{n}, x_{q}\right)-u\left(t_{n}, x_{p}\right)}{d_{p q}}-\nabla u \cdot \mathbf{n}_{\mathbf{p q}}\right)\left(e_{p}^{n+1}-e_{q}^{n+1}\right) d x d t .
\end{aligned}
$$

Substituting these terms into equation (3.5) we obtain:

$$
\begin{aligned}
& \int_{\Omega}\left|\bar{e}^{m}\right|^{2} d x+\sum_{n=0}^{m-1} \int_{\Omega}\left|\bar{e}^{n+1}-\bar{e}^{n}\right|^{2} d x+2 \sum_{n=0}^{m-1} \int_{I_{n}} \sum_{\mathcal{E}} \int_{e_{p q} I} g(|J(u)|) \\
& \times \frac{\left(e_{p}^{n+1}-e_{q}^{n+1}\right)^{2}}{d_{p q}} d x d t=\int_{\Omega}\left|\bar{e}^{0}\right|^{2} d x \\
& +2 \sum_{n=0}^{m-1} \int_{I_{n}} \sum_{p \in \tau_{h}} \int_{p}\left(\frac{u\left(t_{n+1}, x_{p}\right)-u\left(t_{n}, x_{p}\right)}{k}-\partial_{t} u\right) e_{p}^{n+1} d x d t
\end{aligned}
$$




$$
\begin{aligned}
& +2 \sum_{n=0}^{m-1} \int_{I_{n}} \sum_{\mathcal{E}} \int_{e_{p q} I}\left(g_{p q}^{\sigma, n}-g(|J(u)|)\right) \frac{u_{p}^{n}-u_{q}^{n}}{d_{p q}}\left(e_{p}^{n+1}-e_{q}^{n+1}\right) d x d t \\
& +2 \sum_{n=0}^{m-1} \int_{I_{n}} \sum_{\mathcal{E}} \int_{e_{p q} I} g\left(|J(u)|\left(\nabla u \cdot \mathbf{n}_{\mathbf{p q}}-\frac{u\left(t_{n}, x_{q}\right)-u\left(t_{n}, x_{p}\right)}{d_{p q}}\right)\left(e_{p}^{n+1}-e_{q}^{n+1}\right) d x d t\right. \\
& +2 \sum_{n=0}^{m-1} \int_{I_{n}} \sum_{\mathcal{E}} \int_{e_{p q} I} g(|J(u)|) \frac{\left(e_{p}^{n+1}-e_{p}^{n}-\left(e_{q}^{n+1}-e_{q}^{n}\right)\right)}{d_{p q}}\left(e_{p}^{n+1}-e_{q}^{n+1}\right) d x d t,
\end{aligned}
$$

or briefly

$$
\begin{aligned}
& \int_{\Omega}\left|\bar{e}^{m}\right|^{2} d x+\sum_{n=0}^{m-1} \int_{\Omega}\left|\bar{e}^{n+1}-\bar{e}^{n}\right|^{2} d x+2 \sum_{n=0}^{m-1} \int_{I_{n}} \sum_{\mathcal{E}} \int_{e_{p q} I} g(|J(u)|) \\
& \times \frac{\left(e_{p}^{n+1}-e_{q}^{n+1}\right)^{2}}{d_{p q}} d x d t=\int_{\Omega}\left|\bar{e}^{0}\right|^{2} d x+I+I I+I I I+I V .
\end{aligned}
$$

Now we must estimate each of the four terms on the right hand side. The estimation of the first term is different from that given in [2] due to different stability condition (2.1), so further regularity of a solution is required. First we use $L_{\infty}$ stability of discrete and continuous solutions and a Taylor expansion for the inner term. We obtain

$$
\begin{aligned}
I & =2 \sum_{n=0}^{m-1} \int_{I_{n}} \sum_{p \in \tau_{h}} \int_{p}\left(\frac{u\left(t_{n+1}, x_{p}\right)-u\left(t_{n}, x_{p}\right)}{k}-\partial_{t} u\right) e_{p}^{n+1} d x d t \\
& =2 \sum_{n=0}^{m-1} \int_{I_{n}} \sum_{p \in \tau_{h}} \int_{p}\left(\frac{1}{k} \int_{I_{n}}\left(\partial_{t} u\left(s, x_{p}\right)-\partial_{t} u(t, x)\right) d s\right) e_{p}^{n+1} d x d t .
\end{aligned}
$$

Now we estimate $|I|$ from above, as:

$$
\begin{aligned}
& |I| \leq 2\left(\left\|\bar{u}_{h, k}\right\|_{L_{\infty}\left(Q_{T}\right)}+\|u\|_{L_{\infty}\left(Q_{T}\right)}\right) \mid \sum_{n=0}^{m-1} \int_{I_{n}} \sum_{p \in \tau_{h}} \int_{p} \frac{1}{k} \int_{I_{n}} \\
& \times\left(\int_{t}^{s} \partial_{t t} u(z, x) d z+\nabla\left(\partial_{t} u\right)\left(s, x_{p}+\eta\left(x_{p}-x\right)\right)\left|x_{p}-x\right|\right) d s d x d t \mid,
\end{aligned}
$$

for some $\eta \in\langle 0,1\rangle$, or

$$
|I| \leq C \sum_{n=0}^{m-1} \sum_{p \in \tau_{h}} \int_{p} \int_{I_{n}}\left(\left|\partial_{t t} u(z, x) d z d x\right|+h\left|\nabla\left(\partial_{t} u\right)\left(s, x_{p}+\eta\left(x_{p}-x\right)\right)\right|\right) d s d x .
$$

Finally we get the estimate 
$|I| \leq 2 C\left(\left\|\bar{u}_{h, k}\right\|_{L_{\infty}\left(Q_{T}\right)}+\|u\|_{L_{\infty}\left(Q_{T}\right)}\right)\left(k\left\|\partial_{t t} u\right\|_{L_{1}\left(Q_{T}\right)}+h\left\|\nabla\left(\partial_{t} u\right)\right\|_{L_{1}\left(Q_{T}\right)}\right)$.

We estimate the second term II as in [2]. First we get the estimate

$$
\begin{gathered}
\left|g_{p q}^{\sigma, n}-g(|J(u)|)\right|=\left|g\left(\left|\nabla G_{\sigma} * \tilde{u}\left(t_{n}, x_{p q}\right)\right|\right)-g\left(\left|\nabla G_{\sigma} * \tilde{u}(t, x)\right|\right)\right| \\
\leq L_{g}\left|\int_{\mathbb{R}^{d}} \nabla G_{\sigma}\left(x_{p q}-\eta\right) \tilde{u}_{h, k}\left(t_{n}, \eta\right) d \eta-\int_{\mathbb{R}^{d}} \nabla G_{\sigma}(s-\eta) \tilde{u}(t, \eta) d \eta\right| \\
\leq L_{g} \int_{\mathbb{R}^{d}}\left|\nabla G_{\sigma}\left(x_{p q}-\eta\right)-\nabla G_{\sigma}(s-\eta)\right|\left|\tilde{u}_{h, k}\left(t_{n}, \eta\right)\right| d \eta \\
\quad+L_{g} \int_{\mathbb{R}^{d}}\left|\nabla G_{\sigma}(s-\eta)\right|\left|\tilde{u}_{h, k}\left(t_{n}, \eta\right)-\tilde{u}(t, \eta)\right| d \eta .
\end{gathered}
$$

We obtain

$$
\begin{gathered}
\left|g_{p q}^{\sigma, n}-g(|J(u)|)\right| \leq L_{g} B\left(\frac{h}{\sqrt{2}}\left\|D^{2} G_{\sigma}\right\|_{L_{\infty}(\Omega)}\left\|u_{h, k}\right\|_{L_{\infty}\left(Q_{T}\right)} m\left(K_{\sigma}\right)+\left\|\nabla G_{\sigma}\right\|_{L_{\infty}(\Omega)}\right. \\
\left.\times\left(\left(\int_{\Omega}\left|\bar{e}^{n}\right|^{2} d x\right)^{\frac{1}{2}}+\int_{\Omega} \int_{t}^{t_{n}}\left|\partial_{t} u(s, x)\right| d s d x+\sum_{p \in \tau_{h}} \int_{p} \int_{x_{p}}^{x}\left|\frac{\partial u(t, y)}{\partial \mathbf{n}}\right| d y d x\right)\right),
\end{gathered}
$$

where $m\left(K_{\sigma}\right)$ is measure of the compact support $K_{\sigma}, \sigma$ is fixed, $B$ is the estimation for mirror reflexion function. We denote

$$
\begin{aligned}
& C_{3}=2 L_{g} B\left\|D^{2} G_{\sigma}\right\|_{L_{\infty}(\Omega)}\left\|u_{h, k}\right\|_{L_{\infty}\left(Q_{T}\right)} m\left(K_{\sigma}\right), \\
& C_{4}=2 L_{g} B\left\|\nabla G_{\sigma}\right\|_{L_{\infty}(\Omega)}, \quad C_{g} \text { is such that } g(|J(u)|) \geq C_{g} .
\end{aligned}
$$

The last estimate can be established using the properties of the solution $u$. Hence the term $I I$ can be estimated as follows:

$$
\begin{aligned}
& I I \leq C_{3} h\left(\sum_{n=0}^{m-1} k \sum_{\mathcal{E}} \int_{e_{p q} I} \frac{\left|u_{q}^{n}-u_{p}^{n}\right|^{2}}{d_{p q}} d x\right)^{\frac{1}{2}}\left(\sum_{n=0}^{m-1} k \sum_{\mathcal{E}} \int_{e_{p q} I} \frac{\left|e_{p}^{n+1}-e_{q}^{n+1}\right|^{2}}{d_{p q}} d x\right)^{\frac{1}{2}} \\
& +C_{4} \sum_{n=0}^{m-1} k\left(\sum_{\mathcal{E}} \int_{e_{p q} I} \frac{\left|u_{q}^{n}-u_{p}^{n}\right|^{2}}{d_{p q}} d x\right)^{\frac{1}{2}}\left(\sum_{\mathcal{E}_{e_{p q} I}}\left(\frac{\left|e_{p}^{n+1}-e_{q}^{n+1}\right|^{2}}{d_{p q}} d x\right)^{\frac{1}{2}}\left(\int_{\Omega}\left|\bar{e}^{n}\right|^{2} d x\right)^{\frac{1}{2}}\right. \\
& +C_{4} \sum_{n=0}^{m-1} \int\left(\sum_{I_{n}} \int_{e_{p q} I} \frac{\left|u_{q}^{n}-u_{p}^{n}\right|^{2}}{d_{p q}} d x\right)^{\frac{1}{2}}\left(\sum_{\mathcal{E}} \int_{e_{p q} I} \frac{\left|e_{p}^{n+1}-e_{q}^{n+1}\right|^{2}}{d_{p q}} d x\right)^{\frac{1}{2}} \\
& \times\left(\int_{\Omega} \int_{t}^{t_{n}}\left|\partial_{t} u(s, x)\right| d s d x+\sum_{p \in \tau_{h}} \int_{p} \int_{x_{p}}^{x}\left|\frac{\partial u(t, y)}{\partial n}\right| d y d x\right),
\end{aligned}
$$




$$
\begin{aligned}
& I I \leq \frac{4 C_{2} C_{3}^{2} h^{2}}{C_{g}^{2}}+\frac{1}{2} \sum_{n=0}^{m-1} \int_{I_{n}} \sum_{\mathcal{E}} \int_{e_{p q} I} g(|J(u)|) \frac{\left(e_{p}^{n+1}-e_{q}^{n+1}\right)^{2}}{d_{p q}} d x d t \\
& +\frac{4 C_{4}^{2}}{C_{g}^{2}} \sum_{n=0}^{m-1}\left[k \sum_{\mathcal{E}} \int_{e_{p q} I} \frac{\left|u_{q}^{n}-u_{p}^{n}\right|^{2}}{d_{p q}} d x \int_{\Omega}\left|\bar{e}^{n}\right|^{2} d x+\int \sum_{I_{n}} \int_{e_{p q} I} \frac{\left|u_{q}^{n}-u_{p}^{n}\right|^{2}}{d_{p q}} d x\right. \\
& \times\left(\int_{\Omega} \int_{t}^{t_{n}} \mid \nabla \cdot\left(g(|J(u)| \nabla u)\left|d s d x+\sum_{p \in \tau_{h}} \int_{p} \int_{x_{p}}^{x}\right| \frac{\partial u(t, y)}{\partial \mathbf{n}} \mid d y d x\right)^{2}\right] \\
& =\frac{4 C_{2} C_{3}^{2} h^{2}}{C_{g}^{2}}+I I_{1}+I I_{2}+I I_{3},
\end{aligned}
$$

where the inequalities (3.2), (ii) and equation (1.1) have been used. The last term can be estimated using the properties of the exact solution:

$$
\begin{aligned}
I I_{3} & \leq\left(\frac{8 C_{4}^{2} L_{g} C_{2}}{C_{g}^{2}}\left\|D^{2} G_{\sigma}\right\|_{L_{\infty}(\Omega)}\|\nabla u\|_{L_{\infty}\left(I, L_{2}(\Omega)\right)}+\frac{4 C_{4}^{2} C_{2}}{C_{g}^{2}}\|\Delta u\|_{L_{2}\left(I, L_{2}(\Omega)\right)}\right) k \\
& +\left(\frac{8 C_{4}^{2} L_{g} C_{2}}{C_{g}^{2}}\left\|D G_{\sigma}\right\|_{L_{\infty}(\Omega)}\|\nabla u\|_{L_{\infty}\left(I, L_{2}(\Omega)\right)}\right) h .
\end{aligned}
$$

The third term can be estimated as in [2], but here we present a more accurate estimate:

$$
\begin{aligned}
I I I & =2 \sum_{n=0}^{m-1} \int_{I_{n}} \sum_{\mathcal{E}} \int_{e_{p q} I} g(|J(u)|)\left(\frac{u\left(t_{n}, x_{q}\right)-u\left(t_{n}, x_{p}\right)}{d_{p q}}-\nabla u\left(t_{n}, x\right) \cdot \mathbf{n}_{\mathbf{p q}}\right) \\
& \times\left(e_{p}^{n+1}-e_{q}^{n+1}\right) d x d t+2 \sum_{n=0}^{m-1} \int_{I_{n}} \sum_{p \in \tau_{h}} \sum_{q \in N(p)} \int_{e_{p q}} g(|J(u)|) \\
& \times\left(\nabla u\left(t_{n}, x\right) \cdot \mathbf{n}_{\mathbf{p q}}-\nabla u(t, x) \cdot \mathbf{n}_{\mathbf{p q}}\right) e_{p}^{n+1} d x d t=I I I_{1}+I I I_{2} .
\end{aligned}
$$

We get immediately that

$$
\begin{aligned}
I I I_{2} & =2 \sum_{n=0}^{m-1} \int_{I_{n}} \sum_{p \in \tau_{h}} \int_{p}\left(\nabla g(|J(u)|) \nabla u\left(t_{n}, x\right)-\nabla g(|J(u)|) \nabla u(t, x)\right) e_{p}^{n+1} d x d t \\
& =2 \sum_{n=0}^{m-1} \int_{I_{n}} \sum_{p \in \tau_{h}} \int_{p} \int_{t_{n}}^{t} \partial_{t t}(s, x) d s e_{p}^{n+1} d x d t \\
& \leq 2 k\left(\|u\|_{L_{\infty}(\Omega)}+\left\|\bar{u}^{n+1}\right\|_{L_{\infty}(\Omega)}\right)\left\|\partial_{t t} u\right\|_{L_{1}\left(I, L_{1}(\Omega)\right)}=C_{6} k .
\end{aligned}
$$

In order to estimate term $I I I_{1}$ we introduce the following notation 


$$
R_{p q}=\frac{1}{m\left(e_{p q}\right)}\left(-\int_{e_{p q} I} \nabla u\left(t_{n}, x\right) \cdot \mathbf{n}_{\mathbf{p q}}+\frac{u\left(t_{n}, x_{q}\right)-u\left(t_{n}, x_{p}\right)}{d_{p q}} m\left(e_{p q}\right)\right) d x .
$$

Applying the properties of function $g$, this term can be estimated as

$$
\left|I I I_{1}\right| \leq 2 \sum_{n=0}^{m-1} \int_{I_{n}} \sum_{\mathcal{E}} \int_{e_{p q} I}\left|R_{p q}\right|\left|e_{p}^{n+1}-e_{q}^{n+1}\right| d x d t .
$$

Now using the regularity of a weak solution and the well known estimates of the finite volume method (see [4], chapter 3.1.6) we get

$$
\begin{aligned}
\left|I I I_{1}\right| \leq \frac{C}{C_{g}} h^{2} & \sum_{n=0}^{m-1} \int_{I_{n}} \int_{\Omega}(H(u)(z))^{2} d z d t \\
& +\frac{1}{4} \sum_{n=0}^{m-1} \int_{I_{n}} \sum_{\mathcal{E}} \int_{e_{p q} I} g(|J(u)|) \frac{\left(e_{p}^{n+1}-e_{q}^{n+1}\right)^{2}}{d_{p q}} d x d t .
\end{aligned}
$$

Here $|H(u)(z)|^{2}=\sum_{i, j=1}^{d}\left|D_{i} D_{j} u(z)\right|^{2}$ and $D_{i}$ denote the weak derivative with respect to the component $z_{i}$. Since $u \in L_{2}\left(I, W^{2,2}(\Omega)\right)$ we can denote a constant $C_{5}=\frac{C}{C_{g}}\|H(u)\|_{L_{2}\left(Q_{T}\right)}^{2}$ and we have

$$
\left|I I I_{1}\right| \leq C_{5} h^{2}+\frac{1}{4} \sum_{n=0}^{m-1} \int_{I_{n}} \sum_{\mathcal{E}} \int_{e_{p q} I} g(|J(u)|) \frac{\left(e_{p}^{n+1}-e_{q}^{n+1}\right)^{2}}{d_{p q}} d x d t .
$$

Finally we can estimate the last term as follows:

$$
\begin{aligned}
|I V| \leq & \frac{16}{3} \sum_{n=0}^{m-1} \int_{I_{n}} \sum_{\mathcal{E}} \int_{e_{p q} I} g(|J(u)|) \frac{\left(e_{q}^{n+1}-e_{q}^{n}-\left(e_{p}^{n+1}-e_{p}^{n}\right)\right)^{2}}{d_{p q}} d x d t \\
& +\frac{3}{4} \sum_{n=0}^{m-1} \int_{I_{n}} \sum_{\mathcal{E}} \int_{e_{p q} I} g(|J(u)|) \frac{\left(e_{p}^{n+1}-e_{q}^{n+1}\right)^{2}}{d_{p q}} d x d t \\
\leq & \frac{32}{3} \sum_{n=0}^{m-1} \int_{I_{n}} \sum_{\mathcal{E}} \int_{e_{p q} I} \frac{\left(e_{q}^{n+1}-e_{q}^{n}\right)^{2}+\left(e_{p}^{n+1}-e_{p}^{n}\right)^{2}}{d_{p q}} d x d t \\
& +\frac{3}{4} \sum_{n=0}^{m-1} \int_{I_{n}} \sum_{\mathcal{E}} \int_{e_{p q} I} g(|J(u)|) \frac{\left(e_{p}^{n+1}-e_{q}^{n+1}\right)^{2}}{d_{p q}} d x d t \\
\leq & \frac{32}{3} \sum_{n=0}^{m-1} k \sum_{\mathcal{E}}\left(\left(e_{q}^{n+1}-e_{q}^{n}\right)^{2}+\left(e_{p}^{n+1}-e_{p}^{n}\right)^{2}\right) T_{p q}
\end{aligned}
$$




$$
\begin{aligned}
& \quad+\frac{3}{4} \sum_{n=0}^{m-1} \int_{I_{n}} \sum_{\mathcal{E}} \int_{e_{p q} I} g(|J(u)|) \frac{\left(e_{p}^{n+1}-e_{q}^{n+1}\right)^{2}}{d_{p q}} d x d t \\
& \leq \frac{128}{3} \sum_{n=0}^{m-1} \frac{k}{m(p)} \sum_{p \in \tau_{h}}\left(e_{p}^{n+1}-e_{p}^{n}\right)^{2} T_{p q} m(p) \\
& \quad+\frac{3}{4} \sum_{n=0}^{m-1} \int_{I_{n}} \sum_{\mathcal{E}} \int_{e_{p q} I} g(|J(u)|) \frac{\left(e_{p}^{n+1}-e_{q}^{n+1}\right)^{2}}{d_{p q}} d x d t \leq \frac{128 \bar{T}}{3} \frac{k}{m(p)} \\
& \times \sum_{n=0}^{m-1} \int_{\Omega}\left|\bar{e}^{n+1}-\bar{e}^{n}\right|^{2} d x+\frac{3}{4} \sum_{n=0}^{m-1} \int_{I_{n}} \sum_{\mathcal{E}} \int_{e_{p q} I} g(|J(u)|) \frac{\left(e_{p}^{n+1}-e_{q}^{n+1}\right)^{2}}{d_{p q}} d x d t .
\end{aligned}
$$

We now choose the following relation between scale and mesh size

$$
k=\frac{3}{256 \bar{T}} m(p) .
$$

This relation is correct also for $(2.1)$ for some $\xi \in(0,1)$. Putting all these estimates together and taking into account the relation (3.6), we obtain:

$$
\begin{aligned}
& \int_{\Omega}\left|\bar{e}^{m}\right|^{2} d x+\sum_{n=0}^{m-1} \int_{\Omega}\left|\bar{e}^{n+1}-\bar{e}^{n}\right|^{2} d x+\sum_{n=0}^{m-1} \int_{I_{n}} \sum_{\mathcal{E}} \int_{e_{p q} I} g(|J(u)|) \frac{\left(e_{p}^{n+1}-e_{q}^{n+1}\right)^{2}}{d_{p q}} d x d t \\
& \leq 4 \int_{\Omega}\left|\bar{e}^{0}\right|^{2} d x+2\left(\left\|\bar{u}_{h, k}\right\|_{L_{\infty}\left(Q_{T}\right)}+\| u_{L_{\infty}\left(Q_{T}\right)}\right)\left(k\left\|\partial_{t t}\right\|_{L_{1}\left(Q_{T}\right)}+h\left\|\nabla\left(\partial_{t} u\right)\right\|_{L_{1}\left(Q_{T}\right)}\right) \\
& \quad+\left(\frac{4 C_{2} C_{3}}{C_{g}}+2 C_{5}\right) h^{2}+\left(\frac{8 C_{4}^{2} L_{g} C_{2}}{C_{g}^{2}}\left\|D G_{\sigma}\right\|_{L_{\infty}(\Omega)}\|\nabla u\|_{L_{\infty}\left(I, L_{2}(\Omega)\right)}\right) h \\
& +\left(\frac{8 C_{4}^{2} L_{g}}{C_{g}^{2}}\left\|D^{2} G_{\sigma}\right\|_{L_{\infty}(\Omega)}\|\nabla u\|_{L_{\infty}\left(I, L_{2}(\Omega)\right)}+C_{6}+\frac{4 C_{4}^{2} C_{2}}{C_{g}^{2}}\|\Delta u\|_{L_{2}\left(I, L_{2}(\Omega)\right)}\right) k \\
& \quad+\frac{4 C_{4}}{C_{g}}\left(\sum_{n=0}^{m-1} k \sum_{\mathcal{E}} \int_{e_{p q} I} \frac{\left|u_{q}^{n+1}-u_{p}^{n+1}\right|^{2}}{d_{p q}} d x \int_{\Omega}\left|\bar{e}^{n}\right|^{2} d x\right) .
\end{aligned}
$$

We conclude the proof as in [2]: if we realize, that the first term on the right hand side with $\bar{e}^{0}$ is less then $C h$ because of the properties of the exact solution and the definition of $u_{p}^{0}$, we are prepared to use Gronwall's lemma in the following form.

Lemma 3. If $u(t)$ and $v(t)$ are non-negative measurable functions for $t \geq 0$ and $u_{0}$ is a non- negative constant, then the inequality

$$
u(t) \leq u_{0}+\int_{0}^{t} v(s) u(s) d s
$$


implies that

$$
u(t) \leq u_{0} \exp \left(\int_{0}^{t} v(s) d s\right)
$$

To estimate the last term of the previous inequality let us denote for $t \in I_{n}=\left\langle t_{n-1}, t_{n}\right)$

$$
v(t)=\sum_{\mathcal{E}} \int_{e_{p q} I} \frac{\left|u_{q}^{n+1}-u_{p}^{n+1}\right|^{2}}{d_{p q}} d x, \quad u(t)=\int_{\Omega}\left|\bar{e}^{n}\right|^{2} d x .
$$

All the terms on the left hand side of the obtained above inequality are nonnegative, so we can omit all terms except the first and the inequality still will be valid. On the right hand side of this inequality we estimate the first term:

$$
\left.\left|4 \int_{\Omega}\right| \bar{e}^{0}\right|^{2} d x \mid \leq C h^{2}
$$

where $C$ depends only on the measure of $\Omega$ and the norm of initial condition. From now we denote by constant $C$ the estimations of all norms of the exact solution on the right hand side this inequality, so we obtain

$$
\int_{\Omega}\left|\bar{e}^{m}\right|^{2} d x \leq C\left(h^{2}+h+k\right)+\frac{4 C_{4}}{C_{g}}\left(\sum_{n=0}^{m-1} k \sum_{\mathcal{E}} \int_{e_{p q} I} \frac{\left|u_{q}^{n+1}-u_{p}^{n+1}\right|^{2}}{d_{p q}} d x \int_{\Omega}\left|\bar{e}^{n}\right|^{2} d x\right) .
$$

If we use the properties of function $v$ (see relation (ii) of Lemma 1 ), then we obtain the following inequalities

$$
\begin{gathered}
\int_{\Omega}\left|\bar{e}^{m}\right|^{2} d x \leq C\left(h^{2}+h+k\right) \\
\exp \left(\sum_{n=0}^{m-1} k \sum_{\mathcal{E}} \int_{e_{p q} I} \frac{\left|u_{q}^{n+1}-u_{p}^{n+1}\right|^{2}}{d_{p q}} d x\right) \\
\leq C \exp \left(C_{2}\right)\left(h^{2}+k+h\right),
\end{gathered}
$$

where $C$ is a generic constant depending only on domain $\Omega$, time $T$ and some norms of the exact solution.

Theorem 2. Let the relation (3.6) between scale and space discretization be valid. Then the following error estimates for Perona-Malik weak solution and numerical solution obtained via finite volume method hold

$$
\begin{aligned}
& \sum_{n=0}^{N_{\max }} \int_{I_{n}} \int_{\Omega}\left|u\left(t_{n+1}, x\right)-\bar{u}_{h, k}\left(t_{n+1}, x\right)\right|^{2} d x d t \leq C h \\
& \sum_{n=0}^{N_{\max }} \int_{I_{n}} \sum_{e_{p q} I} m\left(e_{p q}\right) d_{p q}\left(\frac{u_{q}^{n+1}-u_{p}^{n+1}}{d_{p q}}-\frac{1}{m\left(e_{p q}\right)} \int_{e_{p q}} \nabla u \cdot \mathbf{n}_{\mathbf{p q}} d x\right)^{2} d t \leq C h .
\end{aligned}
$$


Proof. It is easy to see that

$$
\begin{aligned}
& \sum_{n=0}^{N_{\max }} \int_{I_{n}} \int_{\Omega}\left|u\left(t_{n+1}, x\right)-\bar{u}_{h, k}\left(t_{n+1}, x\right)\right|^{2} d x d t \\
& \quad \leq 2 h^{2}\|\nabla u\|_{L_{2}\left(I, L_{2}(\Omega)\right.}+2 \sum_{n=0}^{N_{\max }} \int_{I_{n}} \int_{\Omega}\left|\bar{e}^{n+1}\right|^{2} d x d t \leq C h,
\end{aligned}
$$

and the first inequality is proved. Now we get

$$
\begin{aligned}
& \sum_{n=0}^{N_{\max }} \int_{I_{n}} \sum_{e_{p q} I} m\left(e_{p q}\right) d_{p q}\left(\frac{u_{q}^{n+1}-u_{p}^{n+1}}{d_{p q}}-\frac{1}{m\left(e_{p q}\right)} \int_{e_{p q}} \nabla u \cdot \mathbf{n}_{\mathbf{p q}} d x\right)^{2} d t \\
& \quad \leq C \sum_{n=0}^{N_{\max }} \int_{I_{n}} \sum_{e_{p q} I} \int_{e_{p q}} g(J(u)) \frac{\left(e_{p}^{n+1}-e_{q}^{n+1}\right)^{2}}{d_{p q}} d x d t+C \sum_{n=0}^{N_{\max }} \int_{I_{n}} d_{p q} \\
& \quad \times \sum_{e_{p q} I}\left(\frac{u\left(t_{n+1}, x_{q}\right)-u\left(t_{n+1}, x_{p}\right)}{d_{p q}}-\frac{1}{m\left(e_{p q}\right)} \int_{e_{p q}} \nabla u \cdot \mathbf{n}_{\mathbf{p q}} d x\right)^{2} d t \leq C h
\end{aligned}
$$

where we have again used the estimate of the finite volume method for the second term.

Remark 1. We note that error estimates in the $L_{2}$ norm for a linear problem and for an exact solution $u \in C^{2}\left(Q_{T}\right)$ are of order $O(h+k)$ (see [4]). The estimates obtained in this paper are only of order $O\left(h^{\frac{1}{2}}\right)$, when the specified above relation between the scale and mesh size is satisfied. We have not obtained such a quality of the estimates due to the lack of regularity of the exact solution and the strong nonlinearity in the elliptic term.

\section{Numerical Example}

The following example shows the work of the algorithm on an image of the size $480 \times 540$. This image represents a good input for this algorithm based on the Perona-Malik equation, since for edges the large gradients are typical and noise can be characterized as having small gradients. The setting of parameters was the following: $K$ in Perona - Malik function $g$ was set to $150, h$ was equal to $1, T$ to 25 and scale step $k$ to 0.011 .

\section{Acknowledgements}

The authors would like to thank the referee for his constructive criticism which helped to improve the clarity of this note. 


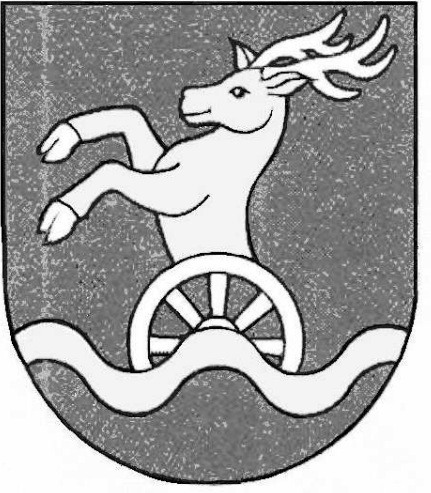

a)

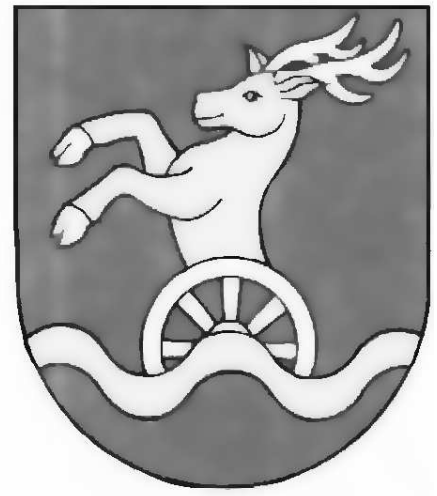

b)

Figure 1. Example. a) the original image, b) the smoothed image.

\section{References}

[1] Z. Krivá. Explicit FV scheme for the Perona-Malik equation. Comput. Methods in Appl. Math., 5(2), 170 - 200, 2005.

[2] A. Handlovičová and Z. Krivá. Error estimates for finite volume scheme for Perona-Malik equation. Acta Math. Unic. Comenianae, 1, 70 - 94, 2005.

[3] F. Catté, P.L.Lions, J.M. Morel and T.Coll. Image selective smoothing and edge detection by nonlinear diffusion. SIAM J. Numer. Anal., 29(1), 182-193, 1992.

[4] R. Eymard, T. Gallouet and R. Herbin. Finite Volume Methods in Handbook of Numerical Analysis 7. Ph. Ciarlet, J.L.Lions (Eds.), Elsevier, 2000.

[5] K. Mikula and N. Ramarosy. Semi-implicit finite volume scheme for solving nonlinear diffusion equations in image processing. Numerische Mathematik, 89(3), $561-590,2001$.

[6] O. A. Ladyženskaja, V. Solonnikov and N.N. Uraľceva. Linear and quasilinear equations of parabolic type. Nauka, Moskva, 1967. (in Russian) 